\title{
Clubfoot pathology in fetus and pathogenesis. A new pathogenetic theory based on pathology, imaging findings and biomechanics- a narrative review
}

\author{
Ernesto Ippolito, Giulio Gorgolini^^
}

Department of Orthopaedic Surgery, University of Rome "Tor Vergata" - Viale Oxford 81, 00133, Rome, Italy

Contributions: (I) Conception and design: E Ippolito; (II) Administrative support: G Gorgolini; (III) Provision of study material or patients: Both authors; (IV) Collection and assembly of data: Both authors; (V) Data analysis and interpretation: E Ippolito; (VI) Manuscript writing: Both authors; (VII) Final approval of manuscript: Both authors.

Correspondence to: Ernesto Ippolito, MD. Professor Emeritus, University of Rome "Tor Vergata” - Viale Oxford 81, 00133, Rome, Italy.

Email: ippolito@med.uniroma2.it.

\begin{abstract}
Several studies have described the pathology of idiopathic congenital clubfoot (ICCF) in fetus. Numerous pathogenetic theories have been postulated on ICCF, but many of them lack any objective evidence. Pathologic studies in fetus together with MRI studies in patients with ICCF seem to favor the theory of a muscular imbalance of the foot activators during fetal growth as the main pathogenetic factor of ICCF. Our objectives were: (I) To support the theory of muscular imbalance as the primary pathogenetic factor of ICCF; (II) To clarify why atrophy and shortening affect the activator muscles of the foot unevenly, as reported by literature. A literature search based on MEDLINE and the COCHRANE database was performed to identify all published studies from 1929 to 2020 which report ICCF pathology in fetus, its etiopathogenesis, and imaging and biomechanical studies showing how the basic pathology may be addressed by Ponseti treatment. A manual search was also performed of the references cited in studies, reviews, and university libraries. Altered size, shape and articular relationships of the tarsal bones, and uneven atrophy and shortening of the leg muscles together with capsule and ligament abnormalities were the main pathologic findings reported in fetus with ICCF. Regarding ICCF pathogenesis, the main debate is between the advocators of a primitive blastemal defect of the tarsal bones leading to the skeletal abnormalities and those who hold that the latter are secondary to a deforming force generated by the soft tissues. Imaging studies have shown that the Ponseti method is able to address the skeletal abnormalities, the correction of which is maintained until adulthood, whereas leg muscle atrophy is not improved but tends to worsen with growth. Preliminary histochemical studies of the soleus-Achilles tendon junction have shown a decrease of the growth factors and the presence of myostatin, both down-regulators of muscle growth in patients with ICCF. The authors postulate that a defect of both the radial and the longitudinal growth unevenly affecting the leg muscles with a consequent imbalance of the foot activators might be the main pathogenetic factor of ICCF. Further studies are needed to confirm this theory.
\end{abstract}

Keywords: Clubfoot; pathology; pathogenesis

Submitted Nov 01, 2020. Accepted for publication Feb 18, 2021.

doi: $10.21037 / \mathrm{atm}-20-7236$

View this article at: http://dx.doi.org/10.21037/atm-20-7236

\footnotetext{
^ ORCID: 0000-0002-0301-9001.
} 


\section{Introduction}

The etiopathogenesis of idiopathic congenital clubfoot (ICCF) is still unknown 2,500 years after its first description by Hippocrates. Regardless of its long-lasting recognition, ICCF etiology is still taking its first steps because-although its genetic origin has been pointed out by clinical studies during the last century (1-4) — the gene(s) responsible for the disease has (have) not yet been identified (5-7). On the contrary, several pathogenetic theories have been proposed (8-15), but many of them are pure conjectures without any anatomical support.

However, several interesting pathologic studies carried out on fetuses and term-stillborns with ICCF have been published during the last 70 years $(11,13-24)$. The detailed description of the virgin and early pathology of both soft tissues and skeletal components of the congenital deformity has allowed us to make new pathogenetic considerations. In addition, MRI studies carried out on newborns and patients with ICCF before and after early treatment by the Ponseti technique (25) have confirmed the pathologic findings observed in fetus, thereby contributing to better highlight its pathogenesis (26-35).

Last but not least, we obtained interesting data from biomechanical studies on the cartilaginous anlagen of the foot $(36,37)$, on the leg muscles $(38,39)$ and on the fibrous tissue of ligaments (40), which are very useful to better understand the mechanisms that produce the typical skeletal deformities in fetus as well as their correction.

The objectives of our narrative review were: (I) To analyze the pathologic studies in fetuses with ICCF, aiming to determine its basic pathology before any treatment; (II) To establish the chronologic sequence of the ICCF pathologic alterations in fetuses of increasing gestational age to better understand the primitive pathogenetic mechanism; (III) To describe the imaging aspects of the bone and soft tissue pathology in newborns with ICCF before treatment and its modification after Ponseti treatment until adulthood; (IV) To postulate a pathogenetic theory of ICCF based on: (i) pathology in fetus; (ii) imaging studies in patients with ICCF treated by the Ponseti method; (iii) biomechanics of foot cartilaginous anlagen, ligaments and leg muscles; (iv) histochemical studies on muscle-tendon units' growth in ICCF.

We present the following article in accordance with the Narrative Review reporting checklist (available at http:// dx.doi.org/10.21037/atm-20-7236).

\section{Methods}

Search strategy and sources of information: The Authors of this review (EI and GG) performed a literature search about the topic by querying online databases. Studies were located by searching the databases Medline (PubMed) and Cochrane Library. The search was performed independently by each author on 1 September 2020 using the following keywords assembled in various combinations to obtain the most pertinent articles: clubfoot, club foot, club-foot, clubfeet, club feet, club-feet, talipes equinovarus, pes equinovarus, pathogenesis, anatomical, embryological, anatomical-physiological, pathological anatomy, embryologic, dissected, anatomical study, functional, histological, histochemical, histochemistry, aetiology, genetic, histopathology, magnetic resonance imaging, magnetic resonance image, MRI, biomechanics. All the keywords were combined to find specific topics about pathology in fetus, ICCF's pathogenesis and MRI and biomechanics in newborns and patients treated by Ponseti method. No language restriction or filter were applied. In addition, a manual search was performed of the references cited in studies, reviews, and pediatric foot and ankle reference textbooks. We also reviewed relevant and pertinent articles from personal and university libraries. Recent review articles on the aforesaid topics were favored.

The two reviewers retrieved the data and independently analyzed each study selected; instances of disagreement were resolved by discussion.

Criteria for inclusion and exclusion: The inclusion criteria were (I) pathology studies carried out only in fetuses and term stillborns with ICCF; (II) most reliable pathogenetic studies providing only a good level of evidence; (III) MRI studies providing only data from mid- to high-field scans in newborns and patients treated by the Ponseti method; (4) all articles regarding the biomechanics of foot cartilaginous anlagen, ligaments and muscles amenable to the Ponseti method. Response letters, comments, case reports, and articles dealing with nonidiopathic clubfoot were excluded.

\section{Discussion}

\section{Patbologic studies in fetus}

We reviewed thirteen pathologic studies carried out in fetuses and stillborns published starting from the second half of the last century (11,13-24). A total of 52 ICCF 
specimens were selected from those studies. The clubfeet were all idiopathic in nature because they belonged to fetuses and term-stillborns in whom no other anomaly had been detected in their musculoskeletal or central nervous systems (arthrogryposis, myelomeningocele, multiple articular deformities, etc.).

Twenty-four ICCF were obtained from fetuses whose gestational age ranged from 12 to 36 weeks; 17 ICCF belonged to term-stillborns, whereas in 11 cases the authors did not specify their exact age, simply saying that the specimens had been obtained either from fetuses of 22-36 weeks of gestational age or from term-stillborns.

There were 25 unilateral cases and 8 bilateral ones, while in 11 fetuses unilateral or bilateral involvement was not specified. In 5 studies, only the skeletal components of the deformity were described $(13,19,21-23)$, three studies prevalently investigated the muscle-tendon units $(16,17,24)$, whereas in 5 studies both the skeletal components and the muscle-tendon units were described $(11,14,15,18,20)$.

Forty-seven ICCF had a gross dissection, while five were studied on serial histologic sections.

\section{Skeletal components}

The main abnormalities have been found in the tarsal skeletal components. Before the completion of their ossification, the skeletal components must be defined as either cartilaginous anlagen in the early stages of foot development or osteocartilaginous anlagen after the appearance of the ossification center. In both the talus and the calcaneus, the ossification center appears at the $24^{\text {th }}-25^{\text {th }}$ week of gestational life, whereas in the cuboid ossification starts at the $36^{\text {th }}$ week (41). All the other tarsal anlagen are still cartilaginous at birth. In this text, we will describe the skeletal components as bones instead of anlagen to make their definition easier. The abnormalities of the ICCF tarsal bones include: (I) alteration of their shape and size; (II) alteration of their articular relationships. However, the skeletal alterations vary with the severity of the ICCF deformity, and they are proportionately greater in termstillborns than in younger fetuses. We believe that this observation is very important because in the unilateral ICCF of the 12-week-old fetus dissected by Bechtol and Mossman (11) - the youngest of the entire series-the prevalent alterations concern the articular relationships of the tarsal bones without anatomical changes. According to the authors' original description: "The skeletal structures of both feet were of the same size and there were no moldings such as a change in the angle of the neck of the talus which is frequently described in older fetuses with clubfoot. The position of the skeletal elements of the foot indicated that three normal joint motions and two abnormal joints motion had taken place. The normal joint motions were: (I) Plantar flexion at the tibio-talar joint; (II) Inversion at the talocalcaneal joint; (III) Cavus deformity or flexion of the entire foot on a transverse axis through all the joints of the arc of the foot. The abnormal motions were: (I) A hinging medially of the calcaneocuboid joint so that the joint was opened laterally as opposed to its usual downward and medial gliding motion. This resulted in the stretching of the lateral ligaments. The navicular was carried to the medial side of the head of the talus. (II) The tarsometatarsal joints were all hinged medially, leading to metatarsus varus deformity."

\section{Alteration of the size and shape of the tarsal bones Talus}

The following changes have been reported in ICCF of fetuses 16-36 weeks old and in term-stillborns. The bone was smaller than normal and misshaped (Figure 1). The trochlea (or dome) was shallower and less convex on the sagittal plane and flat on the transverse plane. It was also uniformly narrower in its transverse diameter. The articular cartilage covered only the posterior part of the trochlea and its upper lateral border was pressed against the fibula, so that the lateral height of the trochlea was somewhat decreased. The fibular articular facet was also enlarged.

Both the neck and the head of the talus were severely angulated medially so that the declination angle of the neck was significantly decreased. In the normal feet, this angle measured $150^{\circ}-155^{\circ}$ whereas in ICCF it measured $115^{\circ}-145^{\circ}$, depending on the severity of the deformity. The neck of the talus was also angulated plantarward up to $50^{\circ}$, whereas in the normal foot the plantar angulation of the neck did not exceed $25^{\circ}-27^{\circ}$. As a consequence, the domeshaped articular surface of the talar head faced medially and inferiorly (Figures 2,3).

\section{Calcaneus}

The calcaneus was smaller than normal. The calcaneal tuberosity was bent medially with an increased convexity of the outer surface of the bone and a corresponding increased concavity of its inner surface.

The anterior process as well as the anterior articular facet for the cuboid were medially twisted. The sustentaculum tali had two articular facets, one for the medial facet of the talus and one for the navicular. 

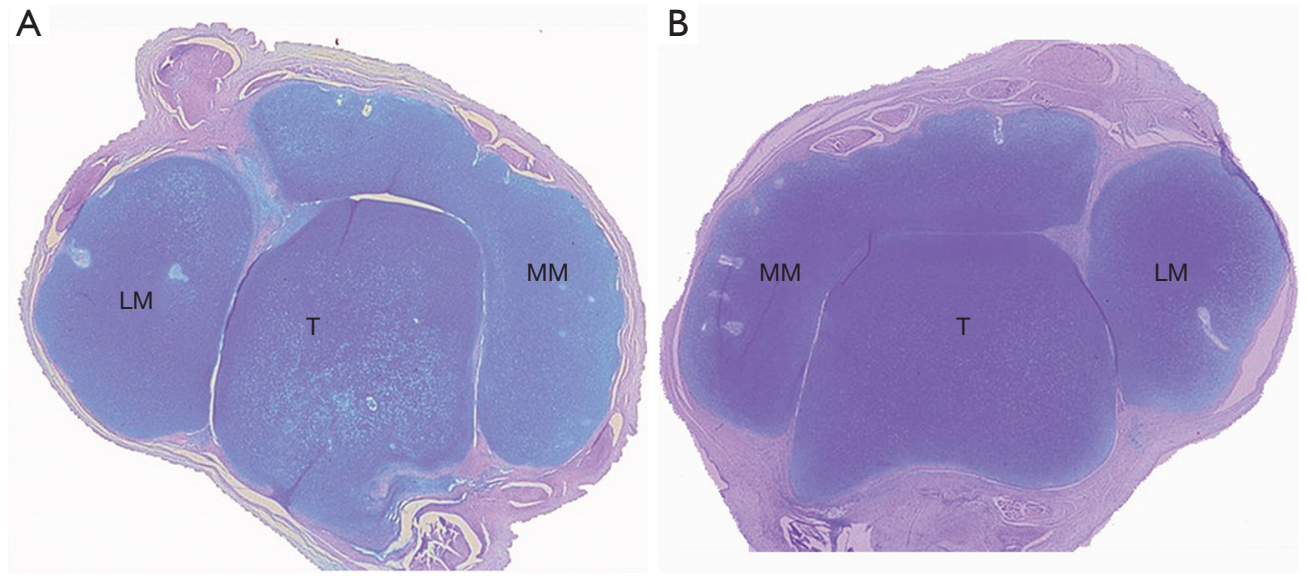

Figure 1 Transverse histological section at the level of the ankle joint of a 16-week-old fetus with a right idiopathic congenital clubfoot (A) and a normal left foot (B). In the clubfoot, the body of the talus (T) is of uniform width and smaller than in the normal foot. Staining method: Alcian Blue - Periodic Acid-Schiff - Hematoxylin; Magnification: ×18. MM, medial malleolus; LM, lateral malleolus.

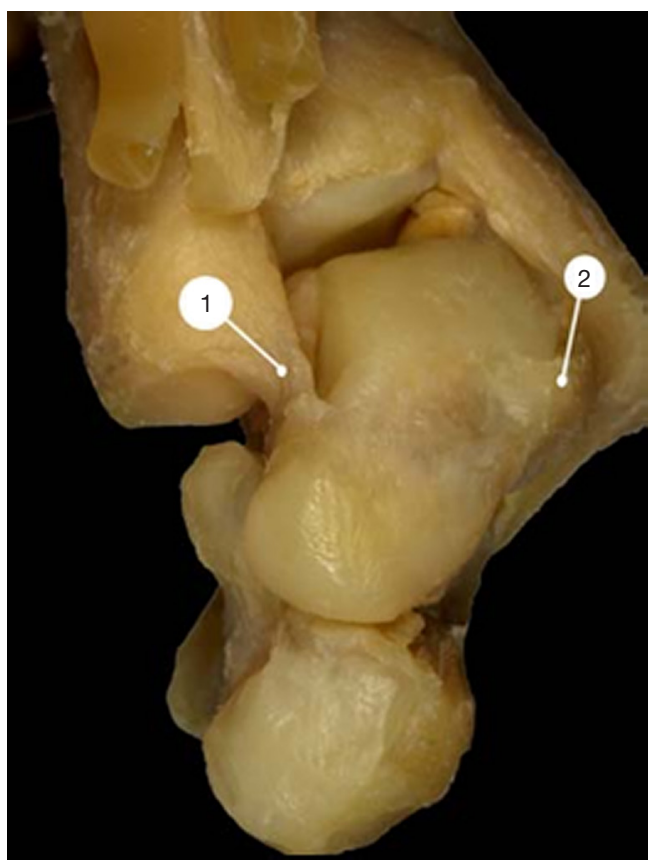

Figure 2 Anterior view of the left idiopathic congenital clubfoot of a fetus aborted after the $25^{\text {th }}$ week of gestation. The ankle joint and the subtalar joint are stretched longitudinally to better show the articular surfaces. The talar neck is markedly deviated medially, and the calcaneus is positioned in varus under the talus. (I) Tibio-talar ligament; (II) Anterior talofibular ligament. (With kind permission from: Windish G. et al. Anatomical Study for an Updated Comprehension of Clubfoot. J Child Orthop 2007;1:79-85).

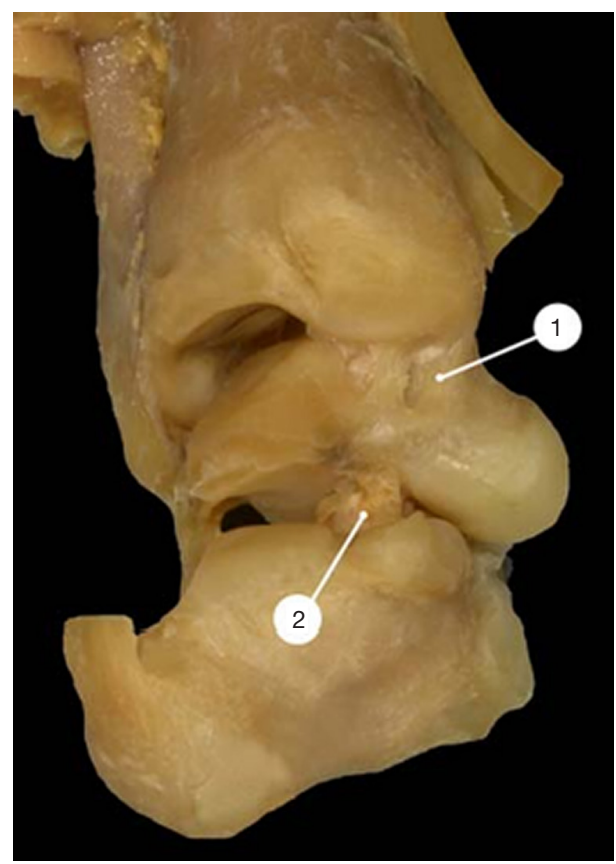

Figure 3 Posteromedial view of the left idiopathic congenital clubfoot of a fetus aborted after the $25^{\text {th }}$ week of gestation. The talar neck is inferiorly angulated in contact with the sustentaculum tali. (I) Tibiotalar ligament; (II) Talocalcaneal interosseous ligament. (With kind permission from: Windisch G. et al. Anatomical Study for an Updated Comprehension of Clubfoot. J Child Orthop 2007;1:79-85). 

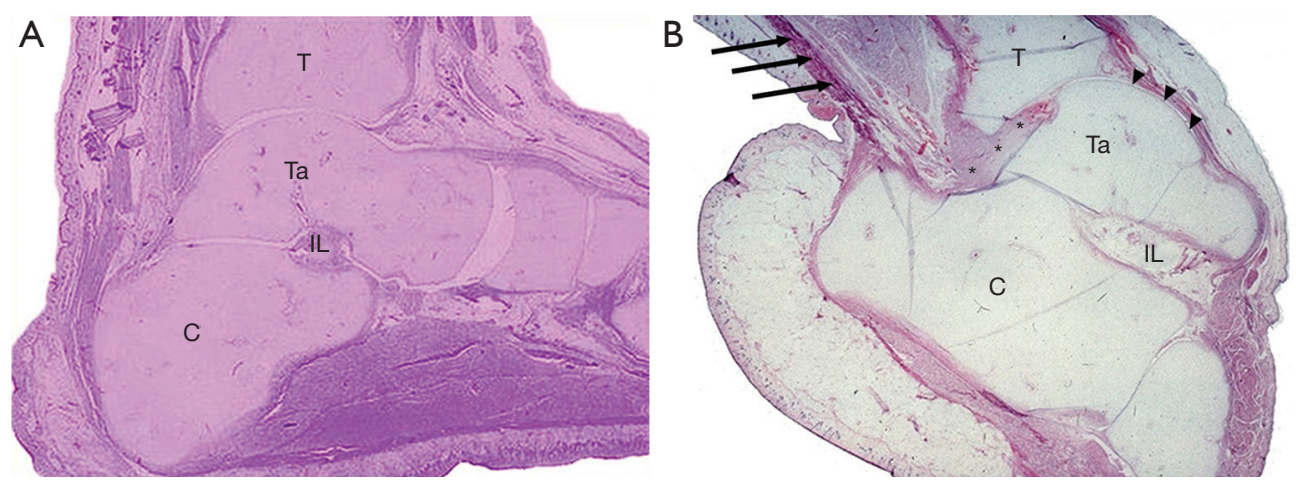

Figure 4 Sagittal histological section at the level of the tibio-talar joint and of the posterior part of the subtalar joint of a 20 -week-old fetus's normal foot (A) and of a 19-week-old fetus's idiopathic congenital clubfoot (B). On the clubfoot side, the talus (Ta) is anteriorly subluxated and the tibia (T) articulates only with the posterior part of the trochlea. The anterior part of the tibio-talar joint capsule is stretched and separated from the anterior articular surface of the trochlea by an open space (arrow heads). On the posterior side, the Achilles tendon is tight (arrows) and the posterior capsule and ligaments of the ankle joint (asterisks) are pulled forward between the non-articular posterior surface of the talus and the inferior articular surface of the tibia. The sinus tarsi is wide and the interosseous talocalcaneal ligament (IL) has a loose structure. Staining method: Hematoxylin-Eosin; Magnification: $\times 10$. C, calcaneus.

\section{Navicular}

The navicular was also smaller than normal. There was an increased curvature of its transverse axis so that the bone was more convex anteriorly and more concave posteriorly than the normal bone. The inner half was larger than the outer, making the bone wedge-shaped.

\section{Cuboid}

No significant structural changes have been described for this bone.

\section{Alteration of the articular relationships of the tarsal bones}

Articular abnormalities have been described for the ankle and all the tarsal joints. As already reported above, in the clubfoot of the youngest fetus dissected by Bechtol and Mossman (11), the ankle and the tarsal joints were subluxated without any noteworthy anatomic alteration of the tarsal bones.

\section{Tibio-talar joint}

The talus was subluxated plantarward in the ankle joint. The anterior part of the trochlea did not articulate with the ankle mortise but was covered by the stretched anterior part of the capsule. As a consequence, the articular surface of the tibia articulated only with the most posterior part of the trochlea (Figure 4).

The posterior ligaments and the posterior capsule of the ankle joint were pulled forward between the posterior surface of the talus and the inferior articular surface of the tibia and were matted together with the posterior ligament of the inferior tibio-fibular syndesmosis (Figure 4).

The Achilles tendon was tight, pulling up the posterior tuberosity of the calcaneus and appearing to be the main cause of the equinus deformity of the hindfoot as shown in the sagittal histologic section of the ICCF of a 19-week-old fetus (14) (Figure 4).

The deltoid ligament was also partly invaginated between the medial malleolus and the medial side of the trochlea as the consequence of the pulling force of the tight tibialis posterior tendon, acting on the medial side of the ankle joint $(14,17,21)$ (Figures 5,6).

\section{Subtalar joint}

The posterior talar facet was triangular-shaped instead of being saddle-shaped as is usual. It was narrowed over its lateral third and flatter than normal.

The groove for the interosseus ligament was shallow and short, and the ligament itself was thin and loose (Figure 4). On the calcaneus side, the posterior and the middle facets were separated by a small and shallow groove for the interosseus ligament in some specimens, whereas in others the two facets were united and the groove for the interosseus ligament was narrow and more laterally located.

The posterior facet extended across the whole width of the talus and calcaneus in the normal foot but only in the inner part of the two bones in ICCF.

The transverse axis of the posterior facet of the subtalar 


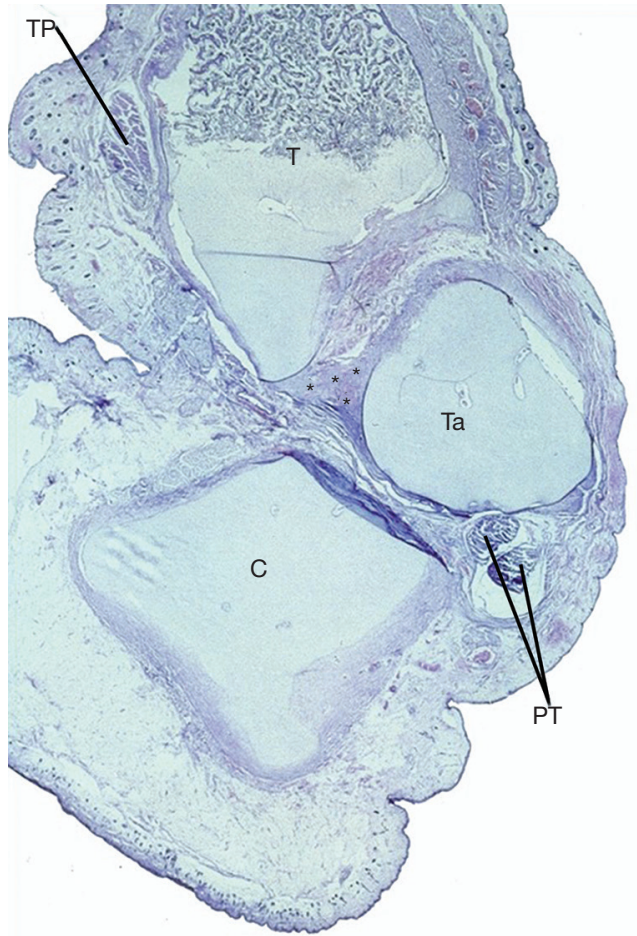

Figure 5 Coronal histological section at the level of the tibio-talar joint and of the anterior part of the subtalar joint of a 19 -week-old fetus's idiopathic congenital clubfoot. The calcaneus (C) is positioned in extreme varus underneath the talus (Ta), and no anterior or middle facets of the subtalar joint are visible. The deep layer of the deltoid ligament is partly infolded into the tibio-talar joint (asterisk). Alcian Blue - Periodic Acid-Schiff - Hematoxylin; Magnification: $\times 10$. TP, tibialis posterior tendon; PT, peroneal tendons.

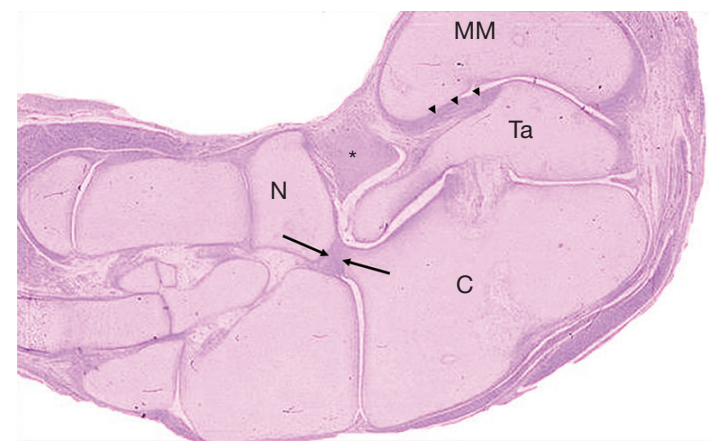

Figure 6 Histological section at the level of the posterior part of the frontal malleolar plane of a 17-week-old fetus's idiopathic congenital clubfoot showing an oblique view of the hindfoot. The tibionavicular ligament merges with the posterior tibial tendon sheath, forming a thick fibrotic mass (asterisk). The deep layer of the deltoid ligament (arrow heads) appears to have been pulled in between the medial malleolus (MM) and the talus (Ta). The plantar calcaneonavicular ligament is very short (arrows). Staining method: Hematoxylin-Eosin; Magnification: $\times 9 . \mathrm{C}$, calcaneus; $\mathrm{N}$, navicular.

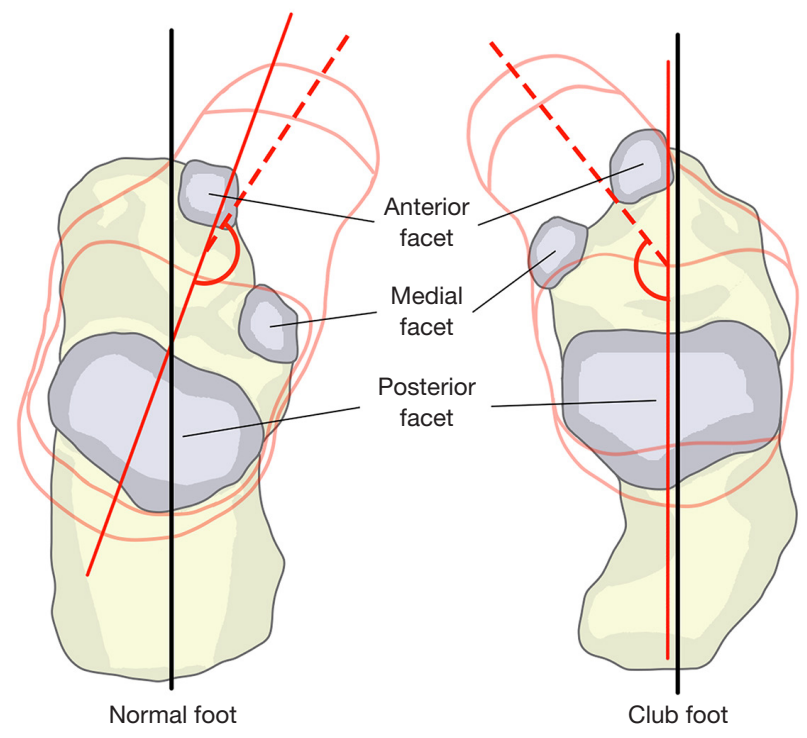

Figure 7 Drawing illustrating the talocalcaneal relationships at the level of the subtalar joint in a pre-term normal foot (left) and clubfoot (right), viewed from the top. In the clubfoot, the calcaneal posterior facet of the subtalar joint is transversally oriented in respect to the longitudinal axis of the calcaneus whereas the middle and the anterior facets adapt their position to the talar neck that is markedly deviated medially; the talus is outlined with a red line: the longitudinal axis of its body (in red) is coincident with the longitudinal axis of the calcaneus (in black) whereas the two axis form the anteriorly open Kite's angle in the normal foot owing to the oblique orientation of the posterior facet in respect to the longitudinal axis of the calcaneus. The dashed line represents the longitudinal axis of the talar neck that forms its declination angle by intersecting the longitudinal axis of the talar body (red line).

joint was oriented at $45^{\circ}$ to the longitudinal axis of the calcaneus whereas the orientation was at $80^{\circ}-90^{\circ}$ in ICCF (Figure 7), thus explaining why the anterior process was directly beneath and in line with the talus. In the normal foot, the anterior and the medial facets supported the head of the talus so that the three facets of the subtalar joint allowed external rotation and pronation of the calcaneus (valgus position) in foot dorsiflexion, while in plantarflexion they allowed internal rotation and supination of the calcaneus (varus position). In ICCF, the transverse orientation of the subtalar joint, together with the medial and plantar angulation of the neck and head of the talus that lie on the postero-medial and anterior facets of the joint, LOCKS the calcaneus in a fixed position of plantarflexion and varus underneath and in line with the talus. 

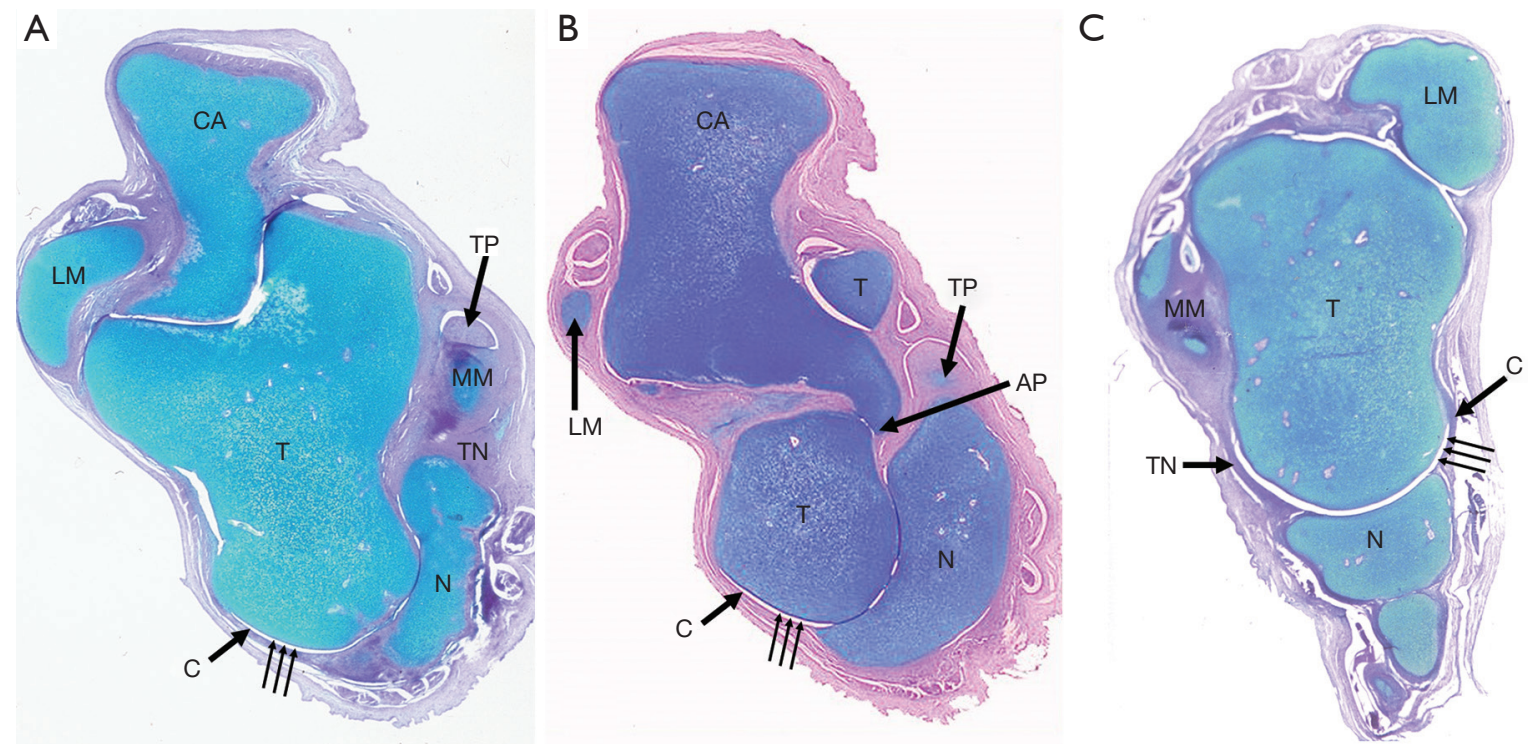

Figure 8 Transverse histological section at the level of the talonavicular joint of the same clubfoot as in Figure 1. (A) Clubfoot; (B) same clubfoot sectioned at a more plantar level; (C) normal foot. In the clubfoot, the navicular (N) is medially and plantarly subluxated on the talar head, and its medial tuberosity is close to the medial malleolus $(\mathrm{MM})$ in $(\mathrm{A})$ and to the anterior process (AP) of the calcaneus in (B). The tibionavicular ligament (TN) is very thick and short on the clubfoot side, while the lateral part of the capsule (C) is stretched and separated from the lateral articular surface of the talar head by an open space (arrows). Staining method: Alcian Blue - Periodic Acid-Schiff - Hematoxylin; Magnification: $\times 12$. T, talus; N, navicular; LM, lateral malleous; CA, calcaneus; TP, tibialis posterior tendon.

\section{Talonavicular joint}

In the ICCF, the joint surface of the head of the talus was dome-shaped and consisted of two parts: the medial part articulated with the navicular that was medially and inferiorly subluxated, whereas the lateral part of the joint formed an open recess covered by the stretched lateral capsule (14) (Figure 8). In older fetuses and term- stillborns, the lateral articular recess was obliterated, and the navicular articulated only with the medial part of the talar head. Owing to its marked medial subluxation apparently due to the tight tibialis posterior tendon, the medial tuberosity of the navicular, together with the insertion of the posterior tibial tendon, were in close contact with the medial malleolus.

The anterior part of the deltoid ligament or tibionavicular ligament was short and thick and merged with the thick sheath of the tibialis posterior tendon and more plantarly with the calcaneonavicular (spring) ligament that was also shortened and thick (Figure 6). The three structures formed a fibrotic mass (Henry's knot) that united the tuberosity of the navicular, the neck of the talus, the medial malleolus and the sustentaculum tali (Figure 9).

\section{Calcaneocuboid joint}

The cuboid was medially subluxated so that only the medial part of the dome-shaped anterior process of the calcaneus articulated with the posterior articular surface of the cuboid (Figure 10).

Both the talonavicular and the calcaneocuboid joints faced medially and plantarward. Since the calcaneus was located medially under the talus, those two joints lay one beneath the other instead of resting approximately side by side as in the normal foot.

\section{Muscles and tendons}

Muscles and tendons were described in detail in only four studies $(17,18,20,24)$. In ICCF, the gastrocnemius-soleus muscle bellies were short and thin, while the Achilles tendon was longer than normal but thinner in some specimens and thicker in some others. The total length of the gastrocnemiussoleus-Achilles tendon was shorter than normal in ICCF, and the difference from normal measurements ranged from $1 \mathrm{~cm}$ in the mild deformities up to $2.5 \mathrm{~cm}$ in the severe ones (Figure 11). The tibialis posterior muscle belly was also small, and its tendon was shorter and thinner than normal, but its insertion on the navicular was thickened. The tendon ran vertically downward, making a groove on the posterior aspect of the medial malleolus from which it could be separated only with difficulty (Figure 12). The total length of the muscle belly 


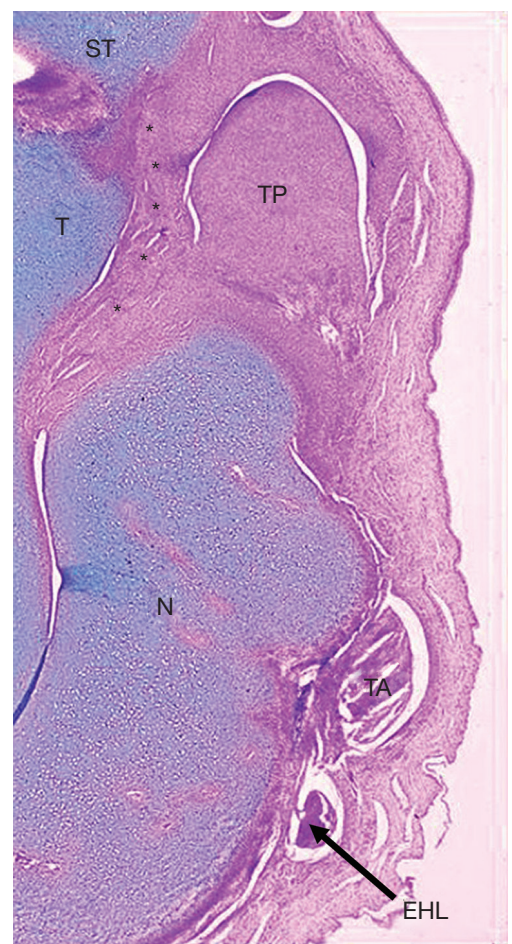

Figure 9 High magnification of a transverse histological section at the level of the plantar aspect of the talonavicular joint of the same clubfoot as in Figures 1 and 8. The insertion of the tibialis posterior tendon (TP) on the navicular $(\mathrm{N})$ is very thick. At this level, the tibionavicular ligament, the thick sheath of the tibialis posterior and the calcaneonavicular ligament form a short and thick fibrotic mass (asterisks) known as Henry's knot that bridges together the navicular, the neck of the talus (T), the sustentaculum tali (ST) and the medial malleolus not visible in this section. The tibialis anterior tendon (TA) appears to be markedly displaced medially, acting as foot supinator rather than as extensor. Staining method: Alcian Blue - Periodic Acid-Schiff - Hematoxylin; Magnification: $\times 300$. EHL, extensor hallucis longus.

and its tendon was shorter than normal, and the difference ranged from a few millimeters up to $2.25 \mathrm{~cm}$ depending on the severity of the deformity. The tibialis anterior and its tendon have been reported to be of normal length in mild cases, a few $\mathrm{mm}$ shorter than normal in moderate cases and up to $1.2 \mathrm{~cm}$ shorter in severe cases $(17,18)$. In ICCF, the tendon of the tibialis anterior ran more medially than normal and close to the tendon of the tibialis posterior before reaching its distal insertion owing to forefoot adduction. The total length of the flexor digitorum longus and its tendon as well as that of the flexor hallucis longus and its tendon were $1.5 \mathrm{~cm}$ shorter in one of Schlicht's (18) specimens. Dietz (15) weighed the

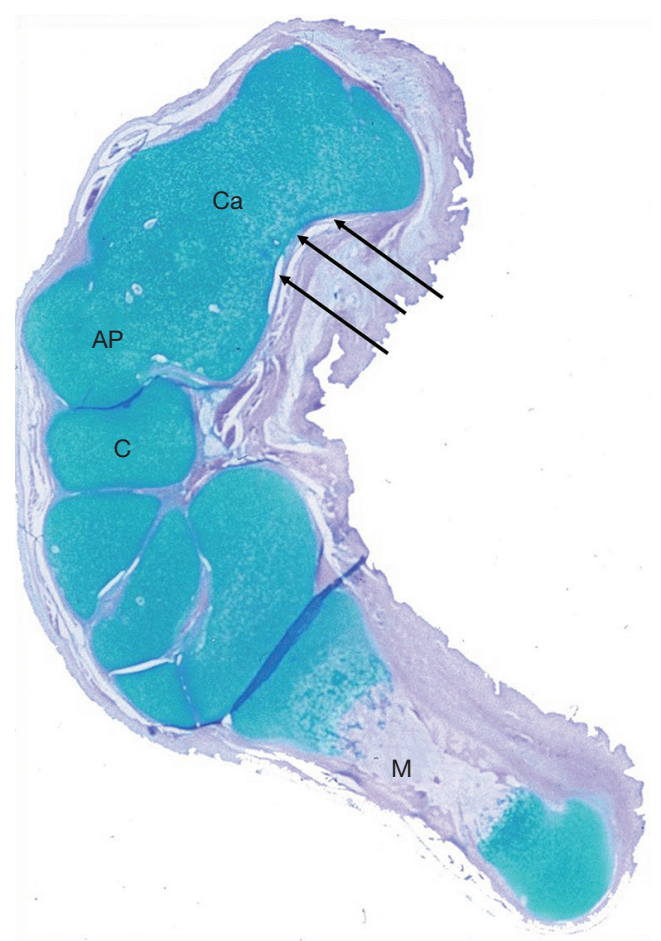

Figure 10 Transverse histological section at the level of the calcaneocuboid joint of the same clubfoot as in Figures 1,8 and 9. The cuboid $(\mathrm{C})$ is medially subluxated on the wedge-shaped anterior process (AP) of the calcaneus (Ca) which is bowed and concave on its medial side (arrows). Staining method: Alcian Blue - Periodic AcidSchiff - Hematoxylin; Magnification: $\times 10$. M, First metatarsal.

leg muscles of a 27 -week-old fetus with unilateral ICCF. The muscles of the postero-medial compartment of the affected leg weighed $67 \%$ less than normal and those of the anterolateral compartment $80 \%$ less than normal.

\section{Functional observations arising from the pathologic studies}

Hjelmstedt and Sarlstedt (21) showed that, after removing the muscles and all the soft tissues but the ligaments around the specimen consisting of the ankle mortise together with the tarsal bones, it was possible by manipulation to obtain only a slight correction of the equinus deformity. The main obstacles were the tight and short ligaments and the medial deviation of the talar neck with the medially subluxated navicular that impinged against the medial malleolus when dorsiflexion was attempted. However, after division of all the ligaments, dorsiflexion of the talus within the malleolar mortise was still obstaculated by the talar deformity itself. Dorsiflexion was then possible only if the talar head was 


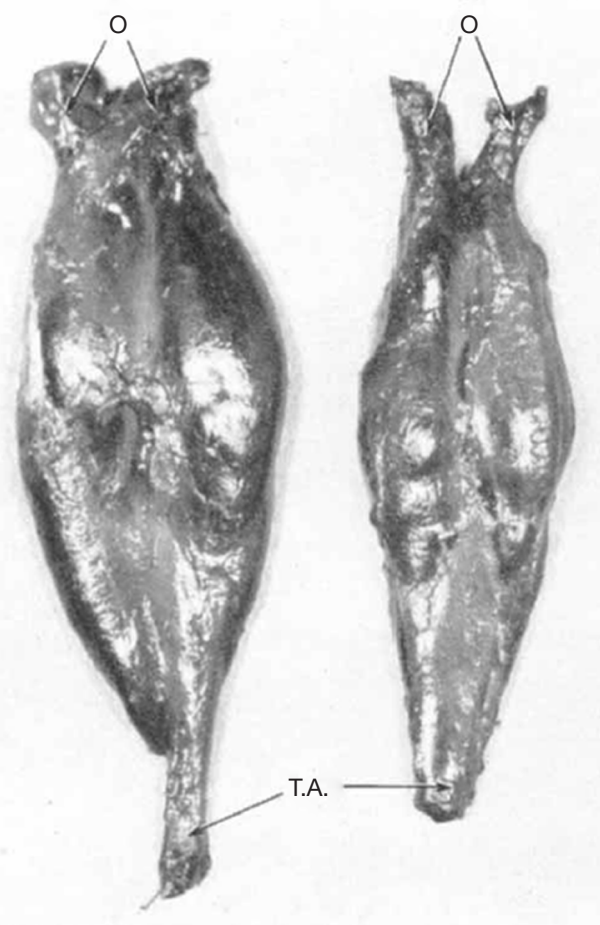

Figure 11 Gastrocnemius, soleus and Achilles tendon dissected from the legs of a term stillborn with a right idiopathic congenital clubfoot. The muscles of the clubfoot leg on the right are thinner and shorter than those of the normal leg on the left. O: Heads of origin of the gastrocnemius; T.A. Achilles tendon severed at the point of insertion into the calcaneus (With kind permission from: Schlicht D. The Pathological Anatomy of Talipes Equinovarus. Austr New Zealand J Surg 1963;33:2-11).

dislocated laterally, thereby losing its contact with the medial malleolus. However, this manoeuvre caused a medial rotation of the posterior part of the trochlea, making the ankle joint incongruent. The plantar deviation of the talar neck also decreased the range of dorsiflexion.

In addition, it is interesting to report the comment of Howard and Benson (22) after the description of the anatomical abnormalities of the subtalar joint: "The malorientation of the subtalar joint articular facets and the deformity of the talar neck cannot be acutely corrected by surgery, but may be molded in a correct anatomical position by serial manipulations and casting that may gradually straighten the talar neck and external rotate the calcaneus to allow a better orientation of the posterior facet of the subtalar joint".

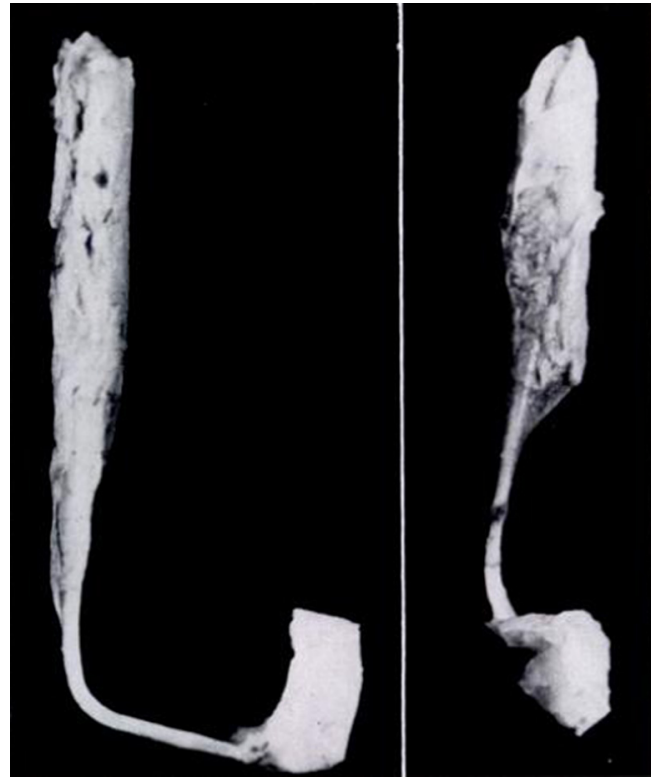

Figure 12 Tibialis posterior and its distal tendon inserting on the medial part of the navicular bone dissected from a fetus with unilateral idiopathic congenital clubfoot. Both the muscle belly and its tendon are markedly shorter on the clubfoot side (right) than on the normal side (left). (With kind permission from: Waisbrod H. Congenital Club Foot: An Anatomical Study. J Bone Joint Surg 1973;55B:796-801).

\section{Imaging studies in patients with ICCF before and after treatment with the Ponseti method}

\section{Skeletal components}

In an MRI study, Pirani et al. (30) showed that Ponseti manipulation and casting treatment (25) in newborns with ICCF can either correct or markedly improve not only the abnormal relationships of the tarsal bones but also their abnormal shape (26-33). That was very likely due to the changes in growth resulting from mechanical stimuli applied to the fast-growing and remodeling fibrous tissue of ligaments (40) and cartilage tissue of tarsal bones $(36,37)$. MRI sequential scans on different spatial planes showed that after 8 weeks of treatment there was either correction or marked improvement of: (I) The tibiotalar plantar flexion (equinus); (II) The abnormal medial (Figure 13) and inferior angulation of the talar neck (adduction and cavus); (III) The dome-shaped deformity of the head of the talus that 

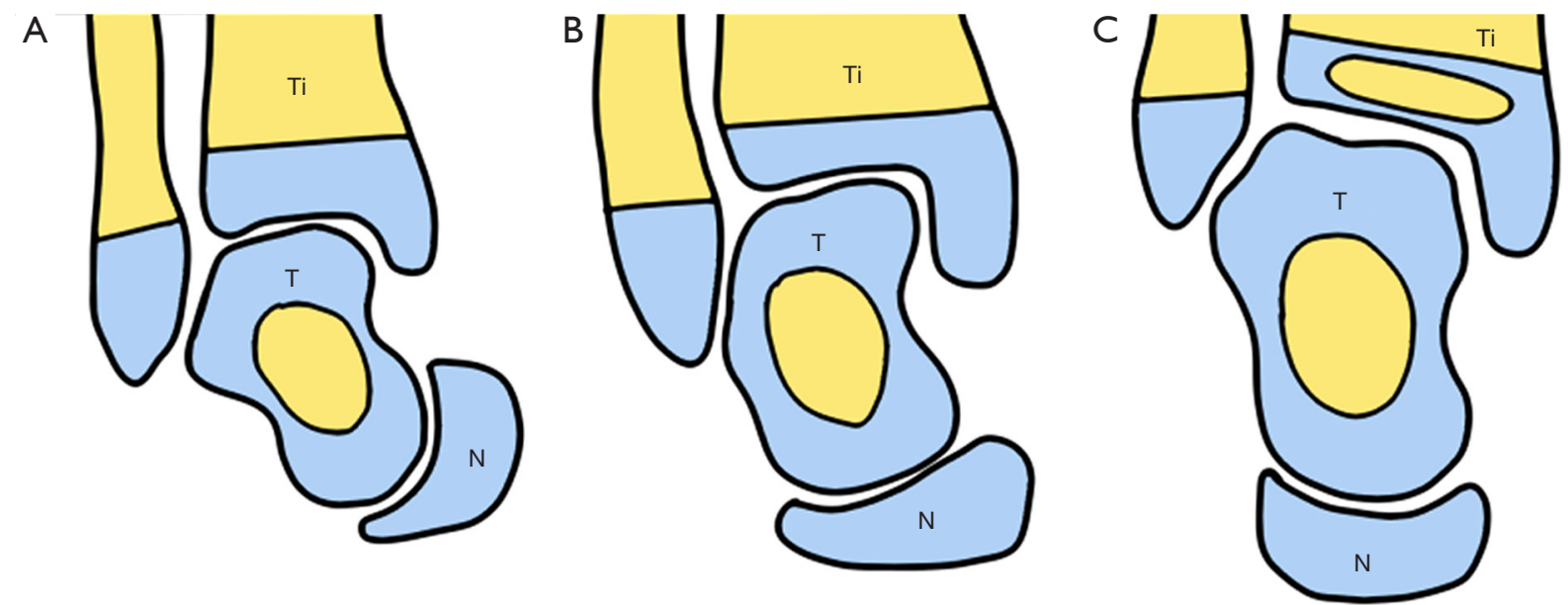

Figure 13 Drawing illustrating how Ponseti manipulation and casting progressively correct within 5 weeks (from A to C) the increased medial angulation of the talar neck, the dome shape of the talar head, the medial talonavicular subluxation and the wedge shape of the tarsal navicular in a two-month-old baby with idiopathic congenital clubfoot. In babies, Magnetic Resonance shows both the cartilaginous (in blue) and the bony (in yellow) components of the osteocartilaginous anlagen of the foot and ankle. T, talus; N, navicular; Ti, tibia.

recovered its spherical shape (Figure 13); (IV) The medial displacement of the navicular (adduction) (Figure 13); (V) The dome-shaped articular part of the anterior process of the calcaneus and the medial-inferior displacement of the cuboid (adduction and cavus); (VI) The altered position of the calcaneus under the talus and of the calcaneocuboid joint that lay under the talonavicular joint instead of laterally to it (varus and supination). Both the recovery and the improvement of the altered articular relationships of the tarsal bones as well as of their altered shape are strictly linked to a recovery of the structural changes of the ligaments observed in fetal ICCF specimens.

\section{Muscles and tendons}

In order to observe the post-natal aspect of muscle-tendon units, MRI studies were also carried out on the leg muscles of unilateral ICCF of untreated newborns and children and adults treated by Ponseti manipulation and casting $(34,35)$. Muscles were measured on transverse MRI scans of both legs taken midway between the articular surface of the knee and the articular surface of the ankle (34).

Measurements of the whole cross-section of the leg were also taken. In untreated newborns, the leg muscles ratio between the normal and the affected side was 1.5 (41.76\% of the normal leg cross-section versus $26.79 \%$ of the ICCF leg), and the total leg surface ratio of the two legs scans was 1.03. In children 2-4 years of age, the leg muscles ratio was 1.8 (42.56\% of the normal leg cross-section versus $22.78 \%$ of the ICCF leg), and the total leg surface ratio of the two legs scans was 1.07. In adults 19-23 years of age, the leg muscles ratio was $2(44.11 \%$ of the normal leg cross-section versus $21.80 \%$ of the ICCF leg), and the total leg surface ratio of the two legs scans was 1.4 (Figure 14).

Those MRI findings confirmed that leg muscle atrophy is present in untreated newborns and is not the consequence of the prolonged treatment to which patients with ICCF are subjected but is rather a primitive component of the congenital deformity, as shown by the pathologic studies in fetuses. Furthermore, muscle atrophy is the only component of the ICCF pathology that tends to worsen with growth even after correction of the deformity, whereas all the other ICCF articular and bony abnormalities maintain their correction until adulthood as shown by imaging studies (42-44).

Another volumetric MRI study in patients with unilateral ICCF ranging in age from 4.8 months to 4.7 years showed a difference in both volume and length between the three muscular compartments of the leg, with the muscles of the ICCF side being thinner and shorter than those of the normal side (35) (Figure 15). The difference between the normal and the ICCF leg muscular compartments was more or less constant until 4-6 years of age, with the exception of the posteromedial muscular compartment in which the difference rose to nine-fold in patients over 1-year-old indicating a marked impairment of muscle growth in that age range. This finding might explain the tendency of ICCF to relapse after 1 year of age, regardless of the treatment 

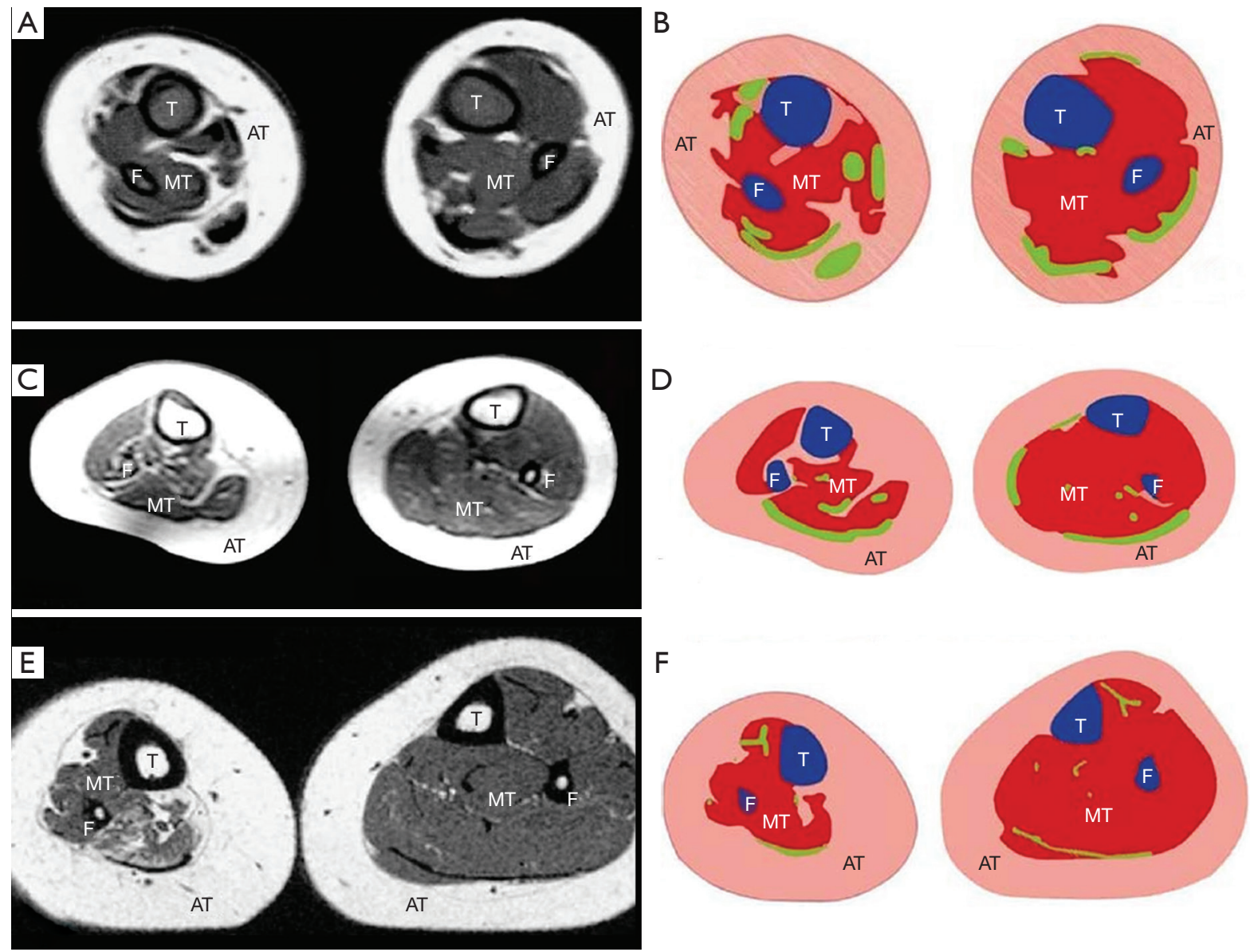

Figure 14 MRI cross scans of the leg patients with congenital clubfoot are shown on the lefts side of the figure whereas on the right side computer elaborations of the same MRI scans are shown. (A) MRI cross scans at the level of the middle third of the legs of a 12-day-old untreated baby with unilateral right idiopathic congenital clubfoot classified as Manes group 3 (Pirani 5-6), showing marked muscle atrophy. (B) Computer elaboration of MRI scans for tissue surface measurement. (C) MRI cross scans of a 4-year-old child, at the same leg level, with unilateral right clubfoot treated by the Ponseti method and classified as Manes group 3 (Pirani 5-6), showing a relative increase of muscle atrophy. (D) Computer elaboration of MRI scan. (E) MRI cross scans of a 20-year-old girl with unilateral right clubfoot treated by the Ponseti method and classified as Manes group 3 (Pirani 5-6), showing a marked increase with age of muscle atrophy (the musculature of the normal leg is almost 3-fold larger than that of the affected leg). (F) Computer elaboration of MRI scans. MT, muscle tissue; AT, adipose tissue; T, tibia; F, fibula.

performed.

\section{Biomechanical studies on foot cartilaginous anlagen and ligaments}

\section{Foot cartilaginous anlagen}

The biomechanical properties of the cartilaginous anlagen of the foot $(36,37)$ which define their compressive behavior and their hydraulic permeability are mainly associated with the collagen network and proteoglycan content.
The collagen network and the cross-linking of its fibrils determine the tensile and shear stiffness of cartilage as well as its compressive properties.

Hydraulic permeability, a measure of the ease of fluid transport throughout the solid matrix, is dependent physically on the porous structure of the matrix and, chemically, on proteoglycan content. The stiffness modulus increases with the increase of the collagen network and the amount of entrapped proteoglycan that retain water with their negative charges. The decrease with age of cartilage 

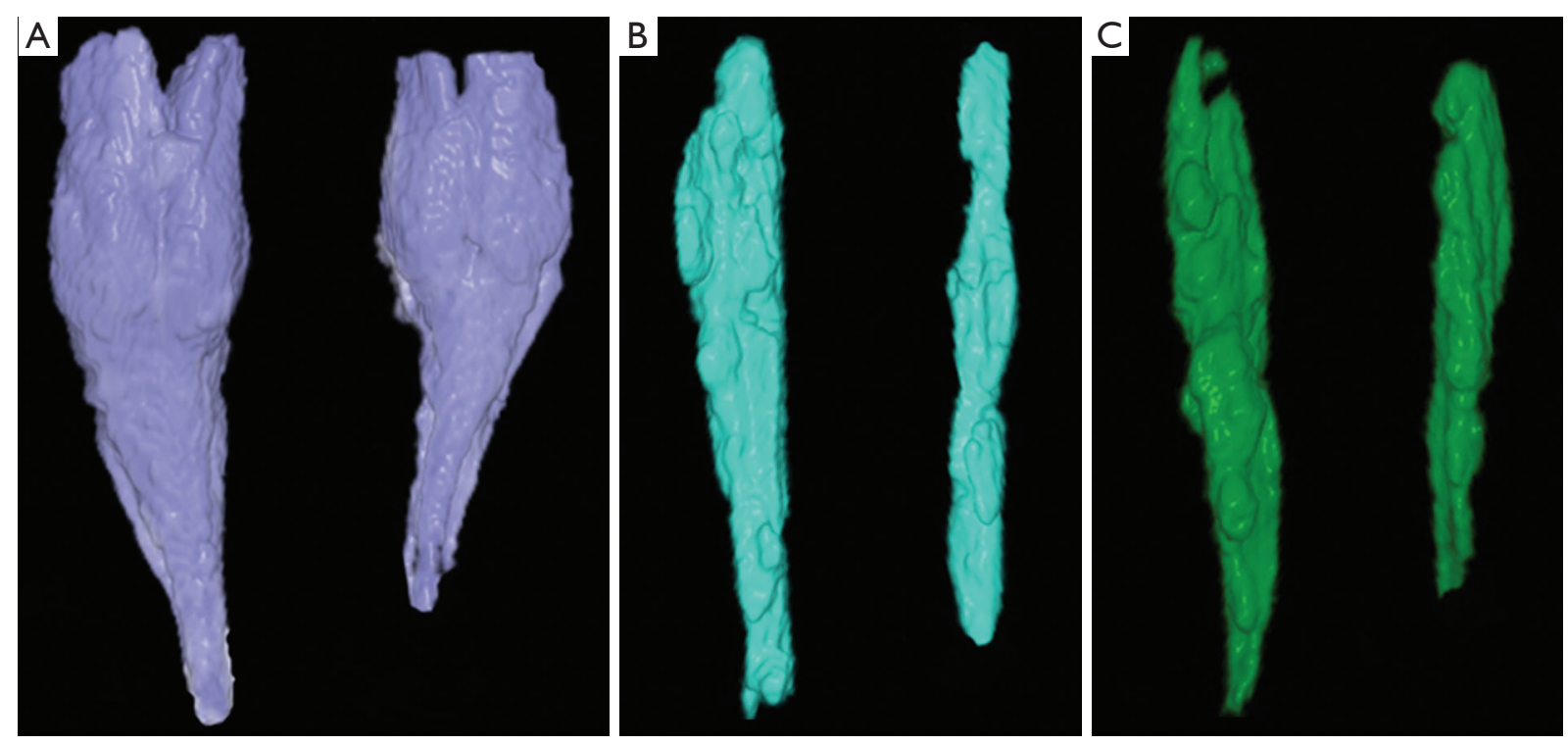

Figure 15 Volumetric magnetic resonance imaging (VMRI) of the posterior (A), anterior (B), and lateral (C) leg muscle compartments of a 4-month-old baby with unilateral right idiopathic congenital clubfoot classified as Pirani 5.5 and treated by the Ponseti method. All the muscle compartments are thinner and shorter than normal on the clubfoot side. Their relatively longer tendons after treatment are not outlined by VMRI.

porosity is due to the increase of the collagen network. This suggests that as cartilage matures, its permeability becomes more sensitive to the quantity of mechanical loading it experiences.

During fetal growth, ICCF cartilaginous anlagen are subjected to deforming forces that cause first subluxation of the ankle and tarsal joints and later alterations of the normal anatomic shape of the tarsal anlagen. According to Hueter-Volkmann (36), cartilage growth is retarded by mechanical compression and accelerated by decompression. When applied to a fast-growing structure rich in cells undergoing high mitotic activity like the fetal cartilaginous anlagen of the ICCF, mechanical stimuli may produce rapid morphologic changes in those structures. Moreover, strong mechanical stimuli may affect the hydraulic permeability of the cartilage and have negative repercussions on its normal growth. After birth, treatment by manipulation and casting aims to reestablish the normal articular relationships of the ankle and tarsal joints and to correct the anatomic deformities of the tarsal anlagen by applying mechanical stimuli that generate plastic deformation of capsules, ligaments and cartilage in the opposite direction of the mechanical stimuli that produced the pathologic changes during the fetal development of the foot (25).

\section{Capsules and ligaments}

The capsules and ligaments of the posteromedial aspect of the foot are relaxed and folded during fetal clubfoot development as shown by pathologic studies $(14,17,21)$. That process implies two events occurring almost simultaneously within the ligaments and capsules fibrous tissue: (I) rearrangement of its microstructure and (II) fast tissue growth (36). The end result of those two events is shortening and thickening of the ligaments with gradual resorption of their part folded into the adjacent joint.

Conversely, the capsules and ligaments of the anterolateral aspect of the foot are stretched and elongated (14) in accordance with the typical stress-strain curve of tendons and ligaments that implies distinct consecutive steps (40). Initially, the wavy collagen structure is stretched out (the "toe region" of the curve). The toe region lies within the elastic limit of the viscoelastic tissue that will return to its original length when unloaded. Further straining elongates the aligned collagen fibers, thereby inducing plastic deformation by collagen interfibrillar sliding caused by breakage of the intermolecular cross-links. In this "linear region" of the curve, the tissue does not return to its original length after unloading (Figure 16).

Here again, the two events of rearrangement of the 


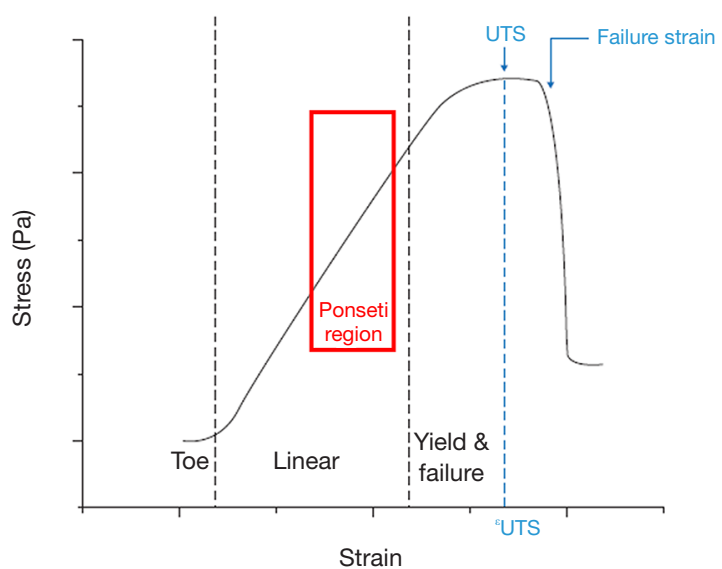

Figure 16 A typical stress-strain curve of a tendon or a ligament. Ultimate tensile strength (UTS) is the maximum stress that a material can withstand before breaking while being tensile loaded. In Ponseti treatment, the stretch caused by manipulation must produce sufficient deformation of clubfoot ligaments for their lengthening and remodeling. Ligament deformation must lie within the middle part of the linear region (plastic deformation-red bracket), since excess deformation would be painful and risk entering the failure region. (With kind permission from: Kadhum et al. An Analysis of the Mechanical Properties of the Ponseti Method in Clubfoot Treatment. Appl Bionics Biomech 2019;2019:4308462).

microstructure of capsules and ligaments and fast tissue growth occur simultaneously during their elongation and thinning. Moreover, if the stretched capsule is pressed against the cartilaginous articular surface of the fetal anlagen, the articular space is obliterated by fibrous adhesions that link the capsule to the articular surface of the fetal anlagen.

When Ponseti manipulation and casting (25) are performed after birth, the capsules and ligaments of the posteromedial and plantar aspect of the foot are elongated, whereas those of the antero-lateral part are folded and shrunk. The biomechanical and metabolic process is, then, the opposite of that causing the deformity in fetus. The Ponseti method is located in the linear region of the stressstrain curve, just before the "failure region" in which the rupture of the ligament occurs (Figure 16).

The same stress-strain curve cannot be applied to the tendons in Ponseti treatment because the latter are stretched together with the muscles that have a different biomechanical behavior.

\section{Pathogenesis}

Many pathogenetic theories have been postulated in ICCF, but some of them have been based on assumptions lacking any objective evidence.

A mechanical theory of compression in utero on the developing foot was proposed by Hippocrates, but the cause of compression was not specified. Denis Browne (10) postulated that diminution of the amniotic fluid as in oligohydramnios prevents foot movements, thus making the foot vulnerable to extrinsic pressure. However, the absence of an increased incidence of ICCF in pregnancies with oligohydramnios seems to negate this theory.

The theory of a neuromuscular defect causing imbalance of extrinsic foot muscles with consequent ICCF deformity was proposed by Dittrich (9). Some morphometric studies found a predominance of type I on type II fibers in the muscles of the posterior compartment of the leg as well as type I fiber grouping and marked variation in fiber size (45-49). However, more recent studies carried out on a large number of ICCF specimens from the same leg compartment found a normal type I to type II fibers ratio $(50,51)$. An electromyographic study carried out in 52 children with congenital clubfoot showed neurogenic electrophysiological patterns in $83 \%$ of the cases, mostly affecting peroneal muscles. The authors' conclusion is that ICCF might be caused by muscle imbalance due to neurologic a bnormality (52). Those data have not been confirmed by further electrophysiological studies on clubfoot muscles.

An arrest of the embryologic foot development was first proposed by Hueter and Von Volkmann and later supported by Böhm (8). However, an embryonic stage of real clubfoot does not exist in normal foot development.

Some authors have proposed that there is a blastemal defect in the development of the tarsal cartilaginous anlagen, causing the anatomic alterations of their shape described in the pathologic studies $(13,19,20,22,23)$. However, those alterations were either minimal or absent in the ICCF of the youngest fetuses $(11,14)$. Moreover, the same anatomical alterations of the tarsal bones have been described in the clubfeet of fetuses affected by muscular imbalance due to a neuromuscular defect like myelomeningocele (19). Lastly, once corrected by treatment, those anatomic alterations do not have the tendency to recur as should be expected in an inborn germ-plast defect (53). In this connection, several diagnostic imaging studies have shown that the correction of the deformities of the cartilaginous tarsal skeletal 
components of ICCF is maintained after the end of skeletal growth (42-44).

A theory of retracting fibrosis involving the soft tissues of the posteromedial aspect of the leg and foot has been supported by the histologic observation of increased fibrous tissue within the ligaments, fasciae muscles and tendon sheaths (14). Myofibroblasts and myofibroblast-like cells have also been found by ultrastructural studies in ligaments and tendon sheaths of patients with ICCF $(54,55)$. Zhao et al. (56) reported an increased content of collagen I, II and III in the clubfeet fasciae in comparison to normal feet of similar age. Poon et al. (57) found high levels of betacatenin-a protein that regulates fibroblastic activityin ICCF fibrotic tissue as well as high levels of type III collagen, thus confirming an increased production of extracellular matrix as in Dupuytren's disease. By contrast, Khan et al. (58) found a few myofibroblast-like cells and normal fibroblasts in capsules, fasciae and ligaments without any difference between the specimens obtained from the lateral and the medial side of the clubfoot and the normal controls. The explanation of those contradictory results may be that the proliferation of fibrous tissue in periarticular soft tissues of ICCF is very active during fetal life and that it decreases gradually during post-natal life (59). A similar behavior has also been described during experimental wound healing (60) in which myofibroblasts are abundant in the early stages of scar tissue formation and then gradually modulate into typical fibroblasts in the late stages when the number of fibroblasts, too, decreases by apoptosis.

Dietz (15) postulated the theory of a regional growth disorder, supported by two clinical observations. First, the leg and foot are smaller than normal in ICCF-the reduction of foot and leg size being more marked in severe deformities-and second, relapse in a corrected clubfoot occurs during the rapid growth period of the foot. The growth disturbance mainly affects the posteromedial side of the leg and foot because both muscles and bones of that region weighed less than those of the antero-lateral side in comparison to normal in a 27 -week-old fetus dissected by the author. He speculated that the regional growth disturbance might be caused by a peripheral nerve abnormality affecting mostly the tibial nerve and to a lesser extent the peroneal nerve. However, the nature of the peripheral nerve abnormality remains obscure.

Abnormalities of tendon insertion on the cartilaginous anlagen of the foot have been proposed by some authors as the cause of the congenital deformity $(12,61)$. However, abnormalities of this kind were not found by any authors at dissection of ICCF fetal specimens.

\section{Theory of muscle imbalance by muscle growth impairment}

The theory of muscle imbalance caused by a non-neurologic etiologic factor that impaired muscle growth was first proposed by Bechtol and Mossman (11), who dissected the unilateral ICCF of a 12-week-old fetus in which the foot deformity was caused only by the subluxation of the tarsal joints without any deformity of the tarsal bones. They did not describe the gross pathology of the dissected muscles of the posteromedial compartment of the leg but, at the microscopic level, they found the presence of "abnormal embryonic muscle fibers". The authors state: "If the abnormal fibers were growing at a retarded rate, they could fail to keep pace with the growth of the skeletal structures, and this would result in their being under tension. The tension could then be the deforming force producing the clubfoot".

This theory was later shared by other authors who reported atrophy and shortening of the leg muscles in fetuses affected by unilateral ICCF, mostly involving the posteromedial compartment of the leg (16-18). Interestingly, in the most severe deformities muscle-tendon units were reported as shorter than in mild and moderate deformities. The advocators of the altered development of the ICCF skeletal components by germ-plast defect $(13,19,20,22,23)$ could object that the muscle-tendon shortening may be the consequence of the primitive skeletal deformities, but some pathologic studies in fetus have demonstrated a different chronologic sequence $(11,14)$. We know from embryologic studies that tarsal cartilaginous anlagen form at six weeks of intrauterine life and that tarsal joints and their ligaments develop at seven weeks, while the activator muscles of the foot form at the eighth-ninth gestational week (41). Histologic as well as some gross pathologic studies have shown that normally developed tarsal joints lost their anatomic relationships as the consequence of an external deforming force $(11,14)$. In fact, both capsule and ligaments were folded into the posterior part of the normal tibiotalar joint, and the anterior capsule appeared to be stretched over the open anterior recess of the joint on the sagittal histologic sections of the ICCF of a 19-weekold fetus. The inner part of the deltoid ligament was also folded between the medial malleolus and its articular facet on the medial side of the talar trochlea $(14,17,21)$ as a result of the combined traction of the Achilles tendon and tibialis posterior tendon that caused varus and supination 


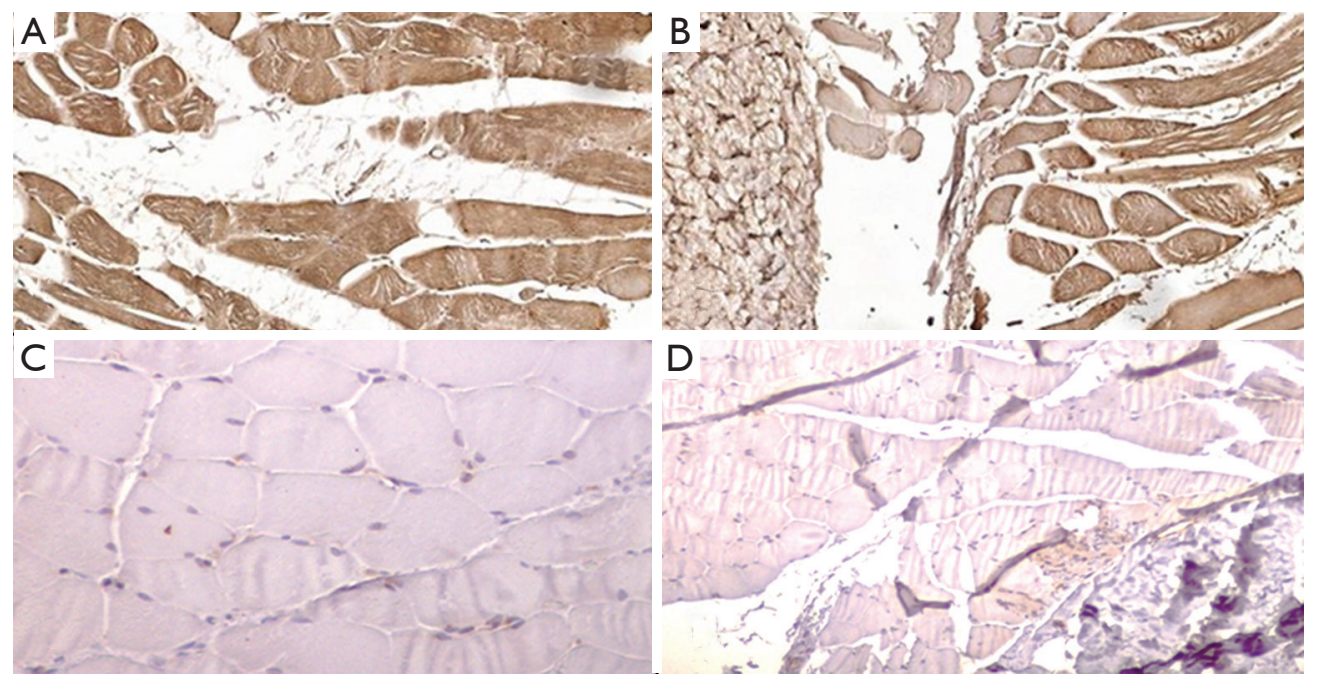

Figure 17 Histological section of the soleus (A) and of the muscle-tendon junction of the soleus-Achilles tendon (B) in a 2-year-old child with a Pirani 5.5 idiopathic congenital clubfoot, and in normal controls (C and D) of a similar age. Histochemical expression of myostatin (brown color in A and B) that inhibits myoblast proliferation and differentiation is marked in the clubfoot but absent in the normal controls. This preliminary study shows that muscle growth is down-regulated in idiopathic congenital clubfoot.

of the tarsal bones. Moreover, on a transversal histologic section at the level of the talonavicular joint of the ICCF of a 16-week-old fetus, the navicular was medially subluxated and separated from the medial malleolus by a thick and folded tibionavicular ligament, while the lateral part of the capsule was stretched over the open lateral recess of the talonavicular joint (14). This means that both joints and capsules were ALREADY NORMALLY developed when the external forces applied by both the Achilles tendon and the tibialis posterior tendon started to exert their traction respectively on the posterior tuberosity of the calcaneus and on the medial tuberosity of the navicular. If the deformity had been caused by a primitive embryologic alteration of the shape and articular relationships of the tarsal skeletal anlagen, the articular space would have been limited to the anatomic borders of the two facing articular surfaces and the ligaments shortened rather than folded into the joint space. In addition, the talar neck appeared to be medially deviated and the head dome shaped as a consequence of the capsular tension.

The combined traction of both triceps surae and tibialis posterior muscles may bring the foot into extreme inversion, i.e., in equinus and varus of the hindfoot and cavus, supination and adduction of the mid-forefoot. The altered pathway of the flexor digitorum communis and of the flexor hallucis longus, both shortened, may in turn further worsen cavus, supination and forefoot adduction (18).
At this point, the question arises: what causes both atrophy and shortening of the muscles of the posteromedial leg compartment and in some cases of the tibialis anterior, too? Dietz (15) postulated that a growth impairment of all the structures of the leg and foot innervated by the tibial and the peroneal nerves might be the cause. In a preliminary histochemical study, we found that some of the growth factors regulating muscle growth were decreased whereas myostatin that inhibits myoblasts proliferation and differentiation was present in the soleus - Achilles tendon junction of patients with ICCF. The same growth factors were normally expressed in controls of a similar age in which myostatin was absent (62) (Figure 17). Therefore, we may assume that an inborn defect of both the radial and the longitudinal growth unevenly affecting the muscles of the leg compartments, could cause the muscular imbalance that in turn causes the clubfoot deformity during fetal development. Further studies on regulating factors of muscle growth are needed in patients with ICCF and in normal subjects of similar age to confirm our theory.

Additional clinical observations that may support our speculation are:

(I) During leg-lengthening, distraction forces may cause leg muscle imbalance that in turn causes foot deformities very similar to ICCF (Figure 18). The imbalance is due to an increased longitudinal growth of the tibialis anterior by stimulation 


\section{Page 16 of 20}

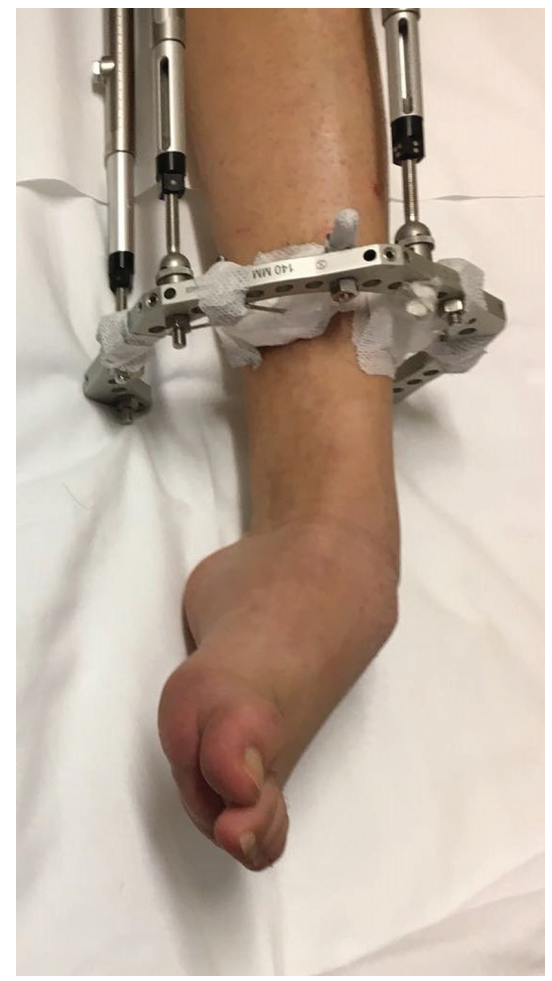

Figure 18 Left leg and foot of an 18-year-old boy who had a $7 \mathrm{~cm} \mathrm{leg-lengthening} \mathrm{at} \mathrm{a} \mathrm{rate} \mathrm{of} 1 \mathrm{~mm}$ per day. At the end of the procedure, a clubfoot-like deformity with claw toes, resistant to manipulative treatment, is evident. The deformity was corrected by lengthening of the Achilles tendon, the tibialis posterior, the flexor digitorum longus and the flexor hallucis longus tendons associated with tibialis anterior tendon transfer to the third cuneiform. The foot deformity was clearly due to muscle imbalance caused by decreased longitudinal growth stimulation of the muscles of the postero-medial compartment of the leg in comparison to the muscles of the antero-lateral compartment.

of myoblasts proliferation as opposed to the gastrocnemius-soleus, the growth in length of which is much less stimulated by the same distracting force, with consequent equinus deformity $(38,39)$. This observation might explain how distraction forces may address longitudinal muscle growth with a similar mechanism in ICCF and explain why, in some cases of ICCF, manipulation and casting are able to obtain equinus correction by stimulating lengthening of the triceps surae, with no need for percutaneous tenotomy of the Achilles tendon. Moreover, the environmental intrauterine factor that controls the rate of the genetic concordance
Ippolito and Gorgolini. Clubfoot pathology and pathogenesis

for ICCF in identical twins $(1,4)$ might be related to the amount of mechanical stimuli to which the leg muscles of the twins are subjected owing to the fetal position in utero and to the amount of central activation of foot movements.

(II) Relapse, a typical characteristic of ICCF, could be explained as arising from a traction imbalance between the less rapid growth of the muscle-tendon units of the postero-medial compartment of the leg compared to the growth of the muscles of the antero-lateral compartment. Relapse occurs most frequently between the first and second year of age, with decreasing frequency up to the age of five or six years and rarely after this age. This parallels the period of rapid foot growth: the foot is $50 \%$ of its adult size by 2.5 years of age and $70 \%$ by 5 years, with relatively slow growth after this time (15). If distraction forces are applied to the leg muscles by a full-time brace up to 1 year of age and thereafter by the body weight of the child standing up during the day and by the brace at night, the relapse rate decreases to $8-9 \%$, but rises to more than $50 \%$ if the brace is abandoned early $(25,63)$. One of the first signs of impending relapse is the so-called dynamic supination of the foot caused by overpulling of the tibialis anterior on its distal insertion on the medioplantar aspect of the foot. This imbalance might be first triggered by a resumption of the impaired longitudinal growth of the tibialis posterior or by an isolated resumption of the impaired growth of the tibialis anterior itself since this muscle is also affected in ICCF and its tendon route is more medially displaced, as shown by both pathologic and MRI studies. However, the foot may be rebalanced and the relapse blocked by the transfer of the tibialis anterior tendon to the third cuneiform, that very likely restores a correct tensioning of the foot activators and stimulates the resumption of their balanced longitudinal growth (63).

\section{Summary}

(I) A genetic influence has been pointed out by epidemiologic studies as an important etiologic factor of ICCF, but no gene(s) responsible for the deformity has (have) yet been identified.

(II) Pathologic studies in fetus have described both skeletal and soft tissue abnormalities, but their 
priority in causing ICCF is still debated.

(VIII) Functional studies on fetal ICCF specimens have shown that some anatomic deformities and some altered articular relationships of the tarsal bones cannot be acutely corrected by surgery because their acute correction produced in turn other articular incongruencies.

(IX) Distraction forces applied to shortened ICCF muscle-tendon units-like those generated by Ponseti treatment-might stimulate myoblast proliferation with consequent recovery of longitudinal muscle growth and lengthening by a mechanism similar to that shown in experimental studies on limb-lengthening.

(X) The same experimental studies have shown that the muscles of the anterior compartment of the leg subjected to distraction forces elongate more than those of the posterior compartment with consequent equinus deformity of the foot. That behavior seems to indicate that there is an inborn tendency of the muscles of the posterior leg compartment to grow in length less than those of the anterior compartment.

(XI) Once obtained, the correction of the skeletal ICCF abnormalities is maintained until adulthood as shown by imaging studies at the end of skeletal growth, whereas muscular atrophy does not improve with growth but worsens in adults, as shown by MRI studies.

(XII) ICCF relapse during the period of maximum foot growth (from 1 to 5 years of age) is best prevented by a brace at night and the body weight of the child when he stands up during the day, since both conditions exert distracting forces on the leg muscles.

(XIII) When ICCF starts to relapse owing to a recurrence of the primitive muscular imbalance, dynamic supination of the foot becomes evident when the child walks. At that stage, tibialis anterior tendon transfer to the third cuneiform blocks relapse, very likely by re-balancing muscle tensioning of the foot activators and in turn stimulating their balanced longitudinal growth.

\section{Acknowledgments}

Funding: None.

\section{Footnote}

Provenance and Peer Review: This article was commissioned by the Guest Editors (Federico Canavese and Alain Dimeglio) for the series "Clubfoot" published in Annals of Translational Medicine. The article has undergone external peer review.

Reporting Checklist: The authors have completed the Narrative Review reporting checklist. Available at http:// dx.doi.org/10.21037/atm-20-7236

Conflicts of Interest: Both authors have completed the ICMJE uniform disclosure form (available at http://dx.doi.org/10.21037/ atm-20-7236). The series "Clubfoot" was commissioned by the editorial office without any funding or sponsorship. The authors have no other conflicts of interest to declare.

Ethical Statement: The authors are accountable for all 
aspects of the work in ensuring that questions related to the accuracy or integrity of any part of the work are appropriately investigated and resolved.

Open Access Statement: This is an Open Access article distributed in accordance with the Creative Commons Attribution-NonCommercial-NoDerivs 4.0 International License (CC BY-NC-ND 4.0), which permits the noncommercial replication and distribution of the article with the strict proviso that no changes or edits are made and the original work is properly cited (including links to both the formal publication through the relevant DOI and the license). See: https://creativecommons.org/licenses/by-nc-nd/4.0/.

\section{References}

1. Idelberger K. Die zwillingspathologie des angeborenen klumpfusses. Z Orthop 1939;(Suppl 69):21-95 (German).

2. Wynne-Davies R. Family studies and the cause of congenital club foot. Talipes equinovarus, talipes calcaneovalgus and metatarsus varus. J Bone Joint Surg Br 1964;46:445-63.

3. Lochmiller C, Johnston D, Scott A, et al. Genetic epidemiology study of idiopathic talipes equinovarus. Am J Med Genet 1998;79:90-6.

4. Engell V, Damborg F, Andersen M, et al. Club foot: a twin study. J Bone Joint Surg Br 2006;88:374-6.

5. Wang LL, Fu WN, Li-Ling J, et al. HOXD13 may play a role in idiopathic congenital clubfoot by regulating the expression of FHL1. Cytogenet Genome Res 2008;121:189-95.

6. Gurnett CA, Alaee F, Desruisseau D, et al. Skeletal muscle contractile gene (TNNT3, MYH3, TPM2) mutations not found in vertical talus or clubfoot. Clin Orthop Relat Res 2009;467:1195-200.

7. Sadler B, Gurnett CA, Dobbs MB. The genetics of isolated and syndromic clubfoot. J Child Orthop 2019;13:238-44.

8. Böhm M. The embryologic origin of club-foot: The Krüppelfürsorgestelle of the City of Berlin in St. Hildegard's Hospital. J Bone Joint Surg 1929;11:229-59.

9. Dittrich R. Pathogenesis of congenital club-foot (pes equinovarus): an anatomical study. J Bone Joint Surg 1930;12:373-99.

10. Browne D. Congenital Deformities of Mechanical Origin: (Section for the Study of Disease in Children). Proc R Soc Med 1936;29:1409-31.

11. Bechtol CO, Mossman HW. Clubfoot; an embryological study of associated muscle abnormalities. J Bone Joint Surg
Am 1950;32 A:827-38.

12. Stewart SF. Club-foot: its incidence, cause, and treatment; an anatomical-physiological study. J Bone Joint Surg Am 1951;33-A:577-90.

13. Irani RN, Sherman MS. The pathological anatomy of club foot. J Bone Joint Surg 1963;45:45-52.

14. Ippolito E, Ponseti IV. Congenital club foot in the human fetus. A histological study. J Bone Joint Surg Am 1980;62:8-22.

15. Dietz FR. Regional growth disorders and the pathogenesis of clubfoot. Iowa Orthop J 1986;6:53.

16. Flinchum D. Pathological anatomy in talipes equinovarus. J Bone Joint Surg Am 1953;35-A:111-4.

17. Wiley A. Club foot: an anatomical and experimental study of muscle growth. J Bone Joint Surg Br 1959;41:821-35.

18. Schlicht $\mathrm{D}$. The pathological anatomy of talipes equinovarus. Aust N Z J Surg 1963;33:1-11.

19. Settle GW. The anatomy of congenital talipes equinovarus: sixteen dissected specimens. J Bone Joint Surg Am 1963;45:1341-54.

20. Waisbrod H. Congenital club foot. An anatomical study. J Bone Joint Surg Br 1973;55:796-801.

21. Hjelmstedt A, Sahlstedt B. Talar deformity in congenital clubfeet. An anatomical and functional study with special reference to the ankle joint mobility. Acta Orthop Scand 1974;45:628-40.

22. Howard CB, Benson M. Clubfoot: its pathological anatomy. J Pediatr Orthop 1993;13:654-9.

23. Windisch G, Anderhuber F, Haldi-Brändle V, et al. Anatomical study for an updated comprehension of clubfoot. Part I: Bones and joints. J Child Orthop 2007;1:69-77.

24. Windisch G, Anderhuber F, Haldi-Brändle V, et al. Anatomical study for an updated comprehension of clubfoot. Part II: Ligaments, tendons and muscles. J Child Orthop 2007;1:79-85.

25. Ponseti IV. Congenital clubfoot: Fundamentals of treatment. Oxford Medical Publication 1996.

26. Grayhack JJ, Zawin JK, Shore RM, et al. Assessment of calcaneocuboid joint deformity by magnetic resonance imaging in talipes equinovarus. J Pediatr Orthop B 1995;4:36-8.

27. Cahuzac JP, Baunin C, Luu S, et Al. Assessment of hindfoot deformity by three-dimensional MRI in infant club foot. J Bone Joint Surg Br 1999;81:97-101.

28. Kamegaya M, Shinohara Y, Kokuji Y, et Al. Evaluation of pathologic abnormalities of clubfoot by magnetic resonance imaging. Clin Orthop Relat Res 
2000;(379):218-23.

29. Kamegaya M, Shinohara Y, Kuniyoshi K, et Al. MRI study of talonavicular alignment in club foot. J Bone Joint Surg Br 2001;83:726-30.

30. Pirani S, Zeznik L, Hodges D. Magnetic resonance imaging study of the congenital clubfoot treated with the Ponseti method. J Pediatr Orthop 2001;21:719-26.

31. Cahuzac JP, Navascues J, Baunin C, et al. Assessment of the position of the navicular by three-dimensional magnetic resonance imaging in infant foot deformities. J Pediatr Orthop B 2002;11:134-8.

32 Saito S, Hatori M, Kokubun S, Abe Y, Kita A. Evaluation of calcaneal malposition by magnetic resonance imaging in the infantile clubfoot. J Pediatr Orthop B 2004;13:99-102.

33 Itohara T, Sugamoto K, Shimizu N, et Al. Assessment of talus deformity by three-dimensional MRI in congenital clubfoot. Eur J Radiol 2005;53:78-83.

34. Ippolito E, De Maio F, Mancini F, et al. Leg muscle atrophy in idiopathic congenital clubfoot: is it primitive or acquired? J Child Orthop 2009;3:171-8.

35. Ippolito E, Dragoni M, Antonicoli M, et al. An MRI volumetric study for leg muscles in congenital clubfoot. J Child Orthop 2012;6:433-8.

36. Brand RA, Siegler S, Pirani S, et al. Cartilage anlagen adapt in response to static deformation. Med Hypotheses 2006;66:653-9.

37. Mahmoodian R, Leasure J, Gadikota H, et al. Mechanical properties of human fetal talus. Clin Orthop Relat Res 2009;467:1186-94.

38. De Deyne PG, Hayatsu K, Meyer R, et al. Muscle regeneration and fiber-type transformation during distraction osteogenesis. J Orthop Res 1999;17:560-70.

39. Hayatsu K, De Deyne PG. Muscle adaptation during distraction osteogenesis in skeletally immature and mature rabbits. J Orthop Res 2001;19:897-905.

40. Kadhum M, Lee MH, Czernuszka J, et al. An Analysis of the Mechanical Properties of the Ponseti Method in Clubfoot Treatment. Appl Bionics Biomech 2019;2019:4308462.

41. Gardner E, Gray DJ, O'Rahilly R. The prenatal development of the skeleton and joints of the human foot. J Bone Joint Surg Am 1959;41-A:847-76.

42. Ponseti IV, El-Khoury GY, Ippolito E, et al. A radiographic study of skeletal deformities in treated clubfeet. Clin Orthop Relat Res 1981;(160):30-42.

43. Ippolito E, Fraracci L, Caterini R, et al. A radiographic comparative study of two series of skeletally mature clubfeet treated by two different protocols. Skeletal Radiol
2003;32:446-53.

44. Ippolito E, Fraracci L, Farsetti P, et al. The influence of treatment on the pathology of club foot. CT study at maturity. J Bone Joint Surg Br 2004;86:574-80.

45. Isaacs $\mathrm{H}$, Handelsman JE, Badenhorst $M$, et al. The muscles in club foot--a histological histochemical and electron microscopic study. J Bone Joint Surg Br 1977;59B:465-72.

46. Handelsman JE, Badalamente MA. Neuromuscular studies in clubfoot. J Pediatr Orthop 1981;1:23-32.

47. Gray DH, Katz JM. A histochemical study of muscle in club foot. J Bone Joint Surg Br 1981;63-B:417-23.

48. Sirca A, Erzen I, Pecak F. Histochemistry of abductor hallucis muscle in children with idiopathic clubfoot and in controls. J Pediatr Orthop 1990;10:477-82.

49. Loren GJ, Karpinski NC, Mubarak SJ. Clinical implications of clubfoot histopathology. J Pediatr Orthop 1998;18:765-9.

50. Maffulli N, Capasso G, Testa V, et al. Histochemistry of the triceps surae muscle in idiopathic congenital clubfoot. Foot Ankle 1992;13:80-4.

51. Herceg MB, Weiner DS, Agamanolis DP, et al. Histologic and histochemical analysis of muscle specimens in idiopathic talipes equinovarus. J Pediatr Orthop 2006;26:91-3.

52. Feldbrin Z, Gilai AN, Ezra E, et al. Muscle imbalance in the aetiology of idiopathic club foot. An electromyographic study. J Bone Joint Surg Br 1995;77:596-601.

53. Shapiro F, Glimcher MJ. Gross and histologic abnormalities of the talus in congenital club foot. J Bone Joint Surg 1979;61:522-30.

54. Zimny ML, Willig SJ, Roberts JM, et al. An electron microscopic study of the fascia from the medial and lateral sides of clubfoot. J Pediatr Orthop 1985;5:577-81.

55. Fukuhara K, Schollmeier G, Uhthoff HK. The pathogenesis of club foot. A histomorphometric and immunohistochemical study of fetuses. J Bone Joint Surg Br 1994;76:450-7.

56. Zhao D, Yue Y, Yang B, et al. Changes of extracellular matrix of congenital clubfoot. J Tissue Eng 2005;9:230-231.

57. Poon R, Li C, Alman BA. Beta-catenin mediates soft tissue contracture in clubfoot. Clin Orthop Relat Res 2009;467:1180-5.

58. Khan AM, Ryan MG, Gruber MM, et al. Connective tissue structures in clubfoot: a morphologic study. J Pediatr Orthop 2001;21:708-12.

59. Sano H, Uhthoff HK, Jarvis JG, et al. Pathogenesis of soft-tissue contracture in club foot. J Bone Joint Surg Br 
Page 20 of 20

$$
\text { 1998;80:641-4. }
$$

60. Darby I, Skalli O, Gabbiani G. Alpha-smooth muscle actin is transiently expressed by myofibroblasts during experimental wound healing. Lab Invest 1990;63:21-9.

61. Inclàn A. Las anomalias de las inserciones tendinosas en la patogenia del piè bot varo equino congenito. Rev Orthop Y Traumatol 1960;5:173 (Spanish).

62. Dragoni M. Fisiopatologia dell'ipotrofia muscolare nel

Cite this article as: Ippolito E, Gorgolini G. Clubfoot pathology in fetus and pathogenesis. A new pathogenetic theory based on pathology, imaging findings and biomechanics-a narrative review. Ann Transl Med 2021;9(13):1095. doi: 10.21037/atm-207236

\section{Ippolito and Gorgolini. Clubfoot pathology and pathogenesis}

piede torto congenito idiopatico. Studio preliminare. PhD Thesis. University of Tor Vergata, Rome, Italy 2016 (Italian).

63. Farsetti P, Caterini R, Mancini F, et al. Anterior tibial tendon transfer in relapsing congenital clubfoot: longterm follow-up study of two series treated with a different protocol. J Pediatr Orthop 2006;26:83-90. 